is naturaleza 
Para la publicación de este número se ha contado con la ayuda

financiera de las siguientes instituciones:

Departamento de Filosofía y Lógica y Filosofía de la Ciencia de la Universidad de Sevilla

Facultad de Filosofía de la Universidad de Sevilla 


\section{NATURALEZA Y LIBERTAD}

Revista de estudios interdisciplinares

Número 4

Málaga, 2014

Esta revista es accesible on-line en el siguiente portal:

http://grupo.us.es/naturalezayl 


\section{Naturaleza y Libertad}

Revista de estudios interdisciplinares

Número 4

ISSN: 2254-96682014

Directores: Juan Arana, Universidad de Sevilla; Juan José Padial, Universidad de Málaga; Francisco Rodríguez Valls, Universidad de Sevilla.

Secretaria: Avelina Cecilia Lafuente, Universidad de Sevilla.

Consejo de Redacción: Jesús Fernández Muñoz, Universidad de Sevilla; José Luis González Quirós, Universidad Juan Carlos I, Madrid; Francisco Soler, Universität Dortmund / Universidad de Sevilla; Pedro Jesús Teruel, Universidad de Valencia; Héctor Velázquez, Universidad Panamericana, México.

Adjunto a la redacción: Miguel Palomo, Universidad de Sevilla

Consejo Editorial: Mariano Álvarez, Real Academia de Ciencia Morales y Políticas; Allan Franklin, University of Colorado; Michael Heller, Universidad Pontificia de Cracovia; Manfred Stöcker, Universität Bremen; William Stoeger, University of Arizona.

Consejo Asesor: Rafael Andrés Alemañ Berenguer. Universidad de Alicante; Juan Ramón Álvarez, Universidad de León; Luciano Espinosa, Universidad de Salamanca; Miguel Espinoza, Université de Strasbourg; Juan A. García González, Universidad de Málaga; José Manuel Giménez Amaya, Universidad de Navarra; Karim Gherab Martín, Urbana University, Illinois; Martín López Corredoira, Instituto de Astrofisica de Canarias; Alfredo Marcos, Universidad de Valladolid; Marta Mendonça, Universidade Nova de Lisboa; Javier Monserrat, Universidad Autónoma de Madrid; Leopoldo Prieto, Colegio Mayor San Pablo, Madrid; Ana Rioja, Universidad Complutense, Madrid. Madrid; José Luis González Recio, Universidad Complutense, Madrid; Javier Serrano, TEC Monterrey (México); Hugo Viciana, Université Paris I; Claudia Vanney, Universidad Austral, Buenos Aires; José Domingo Vilaplana, Huelva.

\section{Redacción y Secretaria:}

Naturaleza y Libertad. Revista de estudios interdisciplinares. Departamento de Filosofia y Lógica. Calle Camilo José Cela s.n. E-41018 Sevilla.

Depósito Legal: MA2112-2012

욜 954.55.77.57 Fax: 954.55.16.78. E-mail: jarana@us.es

(C) Naturaleza y Libertad. Revista de Filosofía, 2014 


\section{ÍNDICE}

\section{ESTUDIOS}

Manuel Alfonseca (Universidad Autónoma de Madrid), Diseño inteligente, evolución al azar, o evolución providencial....... ..11

Paul Gilbert, S J (U. Gregoriana, Roma), Deseo de conocer ..............................................27

Javier Hernández-Pacheco (U. de Sevilla), Evolución, erotismo y origen de las especies. De vuelta desde Darwin a Platón y Aristóteles.

Miguel Ángel Herrero (U. Politécnica de Madrid), La formación de los conceptos científicos. De Grosseteste a Galileo.

Ma Teresa Pelacho López (Zaragoza), Otra autonomía de la ciencia. Reflexiones a la luz del pensamiento de Serguei Kara-murzá.......

Juan Fernando Sellés (U. de Navarra), Estudio de las tesis centrales de Millán-Puelles sobre la libertad....... 189

Francisco Soler Gil (U. de Sevilla), El naturalismo y la tentación de las extrapolaciones omnicomprensivas.

Héctor Velázquez Fernández (U. Panamericana, México), En torno a la naturalización de la conciencia sugerida por Daniel Dennett

\section{SECCIÓN BIBLIOGRÁFICA}

Maria Camila Gallego: Falsos saberes.

Juan Arana: Bernardino Telesio, La naturaleza según sus propios principios. 
Naturaleza y Libertad. Revista de estudios interdisciplinares. Número 4, 2014. ISSN: 2254-9668

\title{
EN TORNO A LA NATURALIZACIÓN DE LA CONCIENCIA SUGERIDA POR DANIEL DENNETT
}

\author{
Héctor Velázquez Fernández \\ Universidad Panamericana, México
}

\begin{abstract}
Resumen: En el pensamiento contemporáneo, Daniel Dennett se ha destacado por hacer ver que ni el materialismo ni el idealismo son vías suficientes para entender la conciencia y su operación. En este texto se expone cómo su modelo de las múltiples versiones permite entender a la conciencia más como una facultad intersubjetiva, combinación de un carácter a la par reductivo y colectivo, que como fruto de una naturalización reduccionista materialista.

Palabras clave: Dennett, conciencia, reduccionismo, anti-reduccionismo, naturalismo. Abstract: In the past decades, Danniel Dennett has been highlighting how neither materialism nor idealism could be accurate ways to understand and explain consciousness. In this text I try to show how his proposal about the multiple versions alouds to understand consciousness more like an intersubjective faculty, than the result of a materialistc reductionist naturalisation.
\end{abstract}

Keywords: Dennett, consciousness, reductionism, antireductionism, naturalism.

Recibido: 18/03/2014. Aprobado: 28/03/2014

\section{BASES PARA UN PROYECTO: PRIMERO EL CONTENIDO Y DESPUÉS LA CONCIENCIA}

Desde finales del siglo XX el estudio de la conciencia han vuelto a ser objeto de renovada investigación científica, junto a una nutrida reflexión filosófica, que ya le había estado dirigiendo su atención desde hace décadas. 
En el marco de esta rehabilitación temática, una de las perspectivas desde la que se la ha abordado, es la llamada teoría cognitivista de la conciencia, que pretende explicarla valiéndose del aparato computacional y representacional en uso dentro de las ciencias cognitivas.

A este proyecto ha contribuido, entre otros, el filósofo norteamericano de orientación empírica, Daniel Dennett.

En los últimos 20 años el pensamiento de Dennett ha sido en reiteradas ocasiones fuente para enfoques que intentan ver en la conciencia uno de los ejemplos más claros para implementar el reduccionismo como método explicativo de la realidad.

Podríamos decir que la propuesta filosófica de Dennett se desarrolla en dos pasos, adelantada por primera vez en la publicación de la tesis que le dirigiera Gilbert Ryle: Content and Consciousness de 1969. Primero, construir una teoría del contenido que sea independiente de la conciencia y más fundamental que ella misma; y después, sobre esta base, desarrollar una teoría de la conciencia entendida como un fenómeno derivado, pero lejos de una visión idealista que la convierta en fundamento de toda intencionalidad o de toda operación mental.

Abordar primero el contenido y después de la conciencia fue una ruta que Dennett ensayó en otras obras posteriores a Contenido y conciencia, como en Brainstorms y en Consciousness Explained; a las que añadió elementos evolucionistas expuestos en Darwin's Dangerous Idea.

La conciencia es para Dennett quizá el último misterio que aún sobrevive entre tantos otros como el del origen del universo o el del origen de la vida, que él ve no como sólo áreas de ignorancia científica sino de completo desconcierto y maravilla. 
Según nuestro autor, aunque el tema de la conciencia deja perplejos por igual al hombre de a pie que al investigador sofisticado, intentar desvelar sus misterios es a veces visto como un atentado, un intento de desmitificación o una suerte de vandalismo intelectual, o hasta un asalto al último santuario de la humanidad, porque pocas facultades parecen tan centrales y personales como ésta.

$\mathrm{Y}$ es que aclarar su estatuto y dar una respuesta a las interrogantes que despierta la conciencia no es sencillo. De entrada, dice Dennett, es natural pensar el yo y su cerebro como dos cosas distintas con diferentes propiedades, peor con estrecha dependencia mutua. Un planteamiento de esta suerte supondría que si el sí mismo es distinto al cerebro, parece que debe estar hecho de una sustancia mental, a manera de la res cogitans de Descartes.

Pues bien, para Dennett hay varias razones para sostener la existencia de esa sustancia mental: la mente consciente no puede ser simplemente el cerebro y ninguna parte propia de él, ya que nada del cerebro podría ser la cosa pensante, la que aprecia una copa de vino, la que desprecia el racismo, recuerda alguna cosa importante o bien obra con responsabilidad moral. Una teoría aceptable de la conciencia humana, dice, debe dar explicación de los motivos o razones expresados al formular los estados mentales.

Esta idea de que la mente sea una entidad separada del cerebro, compuesta de una materia no ordinaria sino de algún tipo de sustancia especial, es una cierta forma de dualismo; que Dennett opone contra el materialismo, para el que solo existe un tipo de sustancia: la material, de la cual se ocupan la física, la química y la fisiología. Para el materialismo la mente sería un fenómeno físico que se explicaría con las mismas leyes y los mismos principios materiales suficientes para dar cuenta de la radiactividad, el movimiento 
Naturaleza y Libertad. Revista de estudios interdisciplinares. Número 4, 2014. ISSN: 2254-9668

de los continentes, la fotosíntesis, la reproducción, la nutrición o el crecimiento. Por lo que excluye principios, reglas o soluciones milagrosas o fuerzas o sustancias o poderes orgánicos inexplicables y desconocidos.

\section{MÁS ALLÁ DEL MATERIALISMO}

Pero nuestro autor, lejos de enfrascarse en inútiles pugnas entre materialismos o idealismos que entiendan la conciencia como una entidad dotada de carácter primario o fundante respecto al resto de la vida psíquica, la intención más bien es buscar instrumentos dentro de las ciencias de la mente que nos permitan apuntalar una fenomenología de la primera persona, como recurso para aclarar cómo funciona la conciencia; una perspectiva de primera persona ante la que se autopresentan los eventos. Es decir, explorar el yo que percibe la realidad que le adviene, como vehículo para entender la conciencia.

Y lo primero es transitar de una mera autofenomenología una heterofenomenología. A lo largo del capítulo 4 de La conciencia explicada, el método de la heterofenomenología es ampliamente descrito como una revolucionaria propuesta acerca de cómo estudiar la conciencia. La idea es apartarse de las ideas del yo y la conciencia expuestas por Husserl y sus seguidores, y en apostar por una heterofenomenología, o minimalismo metafísico, que describa y entienda el mundo del sujeto no como fruto de la introspección, sino como un escenario de objetos varios. Algo así como cuando al explicar un texto, lo analizamos y lo purificamos de todo aquello que pertenezca al yo del autor y genera una ficción teórica más allá del mundo heterofenomenológico de quién lo ha escrito. 
Esto implica abandonar la ruta de la introspección para acceder a la conciencia. Pero Dennett tampoco gusta de la ruta materialista ¿Por qué habría de abandonarse? Piensa que por más sofisticados que sean los materialistas, se olvidan de que una vez omitida la fantasmagórica res cogitans de Descartes no existe una puerta de acceso centralizada o algún centro funcional del cerebro. No sólo la glándula pineal deja de ser la máquina con la cual se envía el fax al alma, como si fuera la oficina presidencial del cerebro, sino que tampoco hay otras partes específicas del mismo cerebro que operen como centro funcional; el cerebro mismo es el cuartel general, el lugar donde reside el observador último, pero no al modo de un cuartel general más profundo o santuario interno, necesario para tener una experiencia consciente; esto es, no existe observador al interior del cerebro.

El rechazo al materialismo se basa en la imposibilidad de que la estructura física justifique la conciencia; y su no aceptación de la autofenomenología del yo se fundamenta en su desconfianza hacia la introspección.

Dennett desacredita los relatos introspectivos porque los considera meros productos verbales o ficciones de utilidad restringida, que no resisten la menor contrastación con resultados empíricos, o la comparación con datos de la psicología social o de la neuropsicología o la psicología del desarrollo (aunque los defensores del ejercicio introspectivo achacan a Dennett no ser del todo anti-introspectivo, porque concede al sujeto cierta forma de autoridad en la explicitación verbal de la experiencia consciente).

¿Qué hacen de la conciencia, pues, la autofenomenología y el anti-introspeccionismo? Según Dennett ambas posturas buscan eliminar la conciencia mediante dos movimientos: primero redefinen la conciencia fenoménica en términos funcionales y representacionales; es decir, la convierten en conciencia 
de acceso: un sistema de automonitoreo del cerebro, por el que los estados de conciencia serían a su vez experiencias de otros estados mentales.

El segundo movimiento, dice Dennett, consiste en negar la existencia de la conciencia fenoménica o al menos las propiedades que se oponen a su naturalización, mediante el rechazo de la distinción consciente/no consciente como vehículo para reflejar la realidad psíquica y sus articulaciones reales.

Así pues, ambos movimientos buscarían la eliminación de la conciencia, y su sustitución por conceptos y distinciones que reflejarían mejor la verdadera naturaleza de la mente. No se trata de un intento por eliminar totalmente la conciencia, sino solo algunas de sus propiedades; es decir, se pretende una eliminación selectiva dentro del marco de una teoría positiva de los aspectos de la conciencia que son juzgados reales.

Y Dennett intenta moverse dentro de ambos movimientos. Su propuesta eliminacionista engloba solo algunas propiedades de la conciencia, como los qualia, o lo que él llama "el teatro cartesiano". Y propone contra ello un modelo bautizado como "múltiples versiones": un representacionismo que analice la conciencia exclusivamente en términos de relaciones de contenido, y al yo como un aspecto emergente de la narración que se construye a través del juego interactivo de los contenidos al interior del sistema.

\section{EL MODELO DE LAS MÚLTIPLES VERSIONES}

Dennett ha desarrollado este modelo de las múltiples versiones a lo largo de más de veinte años. Primero en Contenido y conciencia y luego en la segunda parte de Brainstorms y en una serie de ensayos menos conocidos, que modificó y combinó con nuevos materiales que se convertirían a la postre en 
La conciencia explicada. Que junto a las respuestas a sus críticos recopiladas a lo largo de todos esos años, dieron pie en buena parte a su libro Sweet Dreams.

Uno de los desarrollos subpersonales más importantes de la teoría de la conciencia expuesta por Dennett, es la solución que propone al problema de la temporalización (timing), y al ordenamiento de los objetos en el flujo de conciencia. Esta solución ya tomaba forma en el ensayo Are dreams experiences?, y fue profundizada tanto en Two approaches to mental imagery como en Quining qualia, y alcanza su plena articulación en los capítulos 5 y 6 de $L a$ conciencia explicada.

En esos pasajes, las anomalías temporales de la conciencia (como la referencia al regreso en el tiempo o el retardo subjetivo de la conciencia de la intención) son la base empírica de este modelo de múltiples versiones. En todos estos casos, sostiene Dennett, el materialismo cartesiano no permite discriminar entre la historia narrada por Orwell y otra narrada por Stalin; pues todo lo que tenemos no es más que una diferencia que no hace diferencia. Y él cree que esto confiere plausibilidad a su modelo de las múltiples versiones, dado que niega la existencia de una elaboración inconsciente o preconsciente, y el inicio de la valoración consciente.

A partir de la propuesta de Dennett sobre la naturaleza y la plausibilidad de esta tesis, se ha desarrollado a lo largo de los últimos años un interesante e intenso debate. Las críticas suelen apuntar a la posición verificacionista de Dennett, para el cual, dada la imposibilidad de discriminar entre el relato orwelliano o staliniano, no habría espacio para ninguna verdad (fact of the matter). Otra cuestión muy controvertida es si efectivamente no hay ninguna diferencia, ni siquiera de principio, entre las dos versiones de la realidad. 
Algunos le critican que esta tesis se apoya completamente sobre una posición antirrealista, a lo que Dennett argumenta rechazando la etiqueta de eliminacionista, al aludir a la escala temporal microscópica como la verdadera razón por la cual es imposible distinguir entre una versión orwelliana y staliniana de la realidad. Bien podría uno preguntarse si Dennett es o no un eliminacionista en cuanto a la conciencia fenoménica. Para algunos, la respuesta depende del significado que se atribuya al término qualia. Dennett define los qualia como propiedades inefables, intrínsecas, privativas e inmediatamente accesibles; y de ese modo completa la demolición del sentido cartesiano sostenida por Wittgenstein, Ryle, Austin y Sellars, ya que afirma que no hay posibilidad de realizar una descripción de las propiedades qualia.

De cualquier modo y en concesión a una larga tradición en filosofía y en ciencias sociales, Dennett concibe el lenguaje como un medio para todo el pensamiento conceptual humano. Para este filósofo, la conciencia humana no es un sistema biológico, sino una máquina virtual. Esta máquina sería el producto de comportamientos aceptados o memes que habrían reprogramado nuestros cerebros biológicos. Estos comportamientos de naturaleza lingüística, adquiridos a través de formas de autoestimulación gramatical, actuarían produciendo, repitiendo o reorganizando enunciados en un soliloquio manifiesto o silencioso, como cuando hablamos de nosotros mismos, algo que Dennett considera un lenguaje interno.

Este flujo de verbalización interna habría transformado, según Dennett, la actividad cerebral de los primeros homínidos haciendo que su arquitectura paralela simulase el comportamiento de un operador serial, que a su vez actuara sobre los enunciados del lenguaje natural. Desde este punto de vista, la mente conceptual no sería sino una mera construcción social que se constituye gra- 
cias a la asimilación de los memes por parte de la cultura circundante. Se trata, así, de una versión muy fuerte de la idea según la cual el lenguaje desarrolla un rol constitutivo en el pensamiento.

En La conciencia explicada, Dennett describe qué tipo de programa gira en torno a esta construcción, y lo identifica con el pandemonio de Oliver Selfridge, que hablaba de un modelo de arquitectura computacional, no jerárquica y competitiva, precursor del conexionismo. El pandemonio poseía dos características importantes que Dennett destaca: primero, su arquitectura es un paralelismo elevado y no tiene necesidad de postular un ejecutor central; y en segundo lugar, implica la descomposición funcional recursiva como requisito indispensable al momento que en el teatro cartesiano se anida un súper-homúnculo del cual librarse.

Más recientemente, Dennett ha examinado también otra arquitectura funcional compatible con el modelo de su propuesta de las múltiples versiones: esto es, la teoría de la conciencia entendida como espacio de trabajo global, propuesta por Baars. Según esta teoría, la conciencia es realizada por una sociedad de especialistas, y sostenida por una memoria de trabajo denominada "espacio de trabajo global", cuyos contenidos pueden ser transmitidos al sistema de modo completo.

Dennett ha destacado varios puntos en común entre esta teoría y su modelo de las múltiples versiones. Y en primer lugar, en cuanto a lo que llama el paralelismo elevado; según el cual es la accesibilidad de los especialistas, y no cualquier imaginario alto dirigente o ego central, el que guía desde el principio la explicación de los espectaculares incrementos de competencia cognitiva que asociamos con la conciencia. 
Y esta idea, para Dennett, es el centro de lo que llama el modelo de las múltiples versiones, que asocia a la teoría del espacio del trabajo global mediante la metáfora de la fama del cerebro o la celebridad cerebral. De acuerdo con esta metáfora, la conciencia, al igual que la fama, no es una propiedad intrínseca de los procesos cerebrales, sino que es más bien similar al peso político u otros reconocimientos ajenos hipostasiados.

\section{VENTAJAS DE UNA SANA NATURALIZACIÓN DE LA CONCIENCIA}

¿Qué valoración puede merecer la propuesta naturalista de la conciencia llevada a cabo por Dennett? Si bien es cierto que su teoría de la libertad esta enraizada en la más cruda y primitiva de las concepciones acerca del determinismo físico de la conducta, creo que su teoría acerca de la conciencia presenta varios elementos nada despreciables al momento de intentar comprender cómo es que el ser humano elabora, conserva y comunica sus estados mentales.

Es verdad que los dualismos en ningún sentido parecen operativos, pero no es menos cierto que los monismos materialista o espiritualista resuelven insuficientemente la dinámica de la mente humana, pues uno depende de desdeñar resortes tan íntimamente humanos como las aspiraciones, las convicciones, o los deseos, reduciéndolos a meros mecanismos funcionales de supervivencia en el entorno; mientras que el otro cierra su consideración a los elementos bioquímico mecánicos que actúan para materializar en acciones concretas los resultados de los pensamientos y el lenguaje.

La ventaja del planteamiento de este autor es que convierte la conciencia en un mecanismo que no opera como fundamento metafísico idéntico y por 248 
igual para todos los seres humanos; como si existiera una suerte de nube de la cual abrevaran todos los estados mentales de la humanidad. Quita lo fantasmal a la conciencia para hacerlo al mismo tiempo construcción y potencia, facultad y resultado. Porque si bien es cierto que una de sus fuentes es la introspección, no lo es menos que el escenario de su manifestación es la comunidad con otros.

Y creo que en este punto puede radicar, si se me permite el término, lo que podríamos llamar una sana naturalización. Esto es, aquella que reconoce que para fenómenos que nacen de la percepción física individual y concreta de los sentidos particulares, sólo cabe la identificación concreta y particular de esa experiencia material que nace de la sensibilidad y pasa a la mente del sujeto. Creo que en ese enfoque la naturalización de la conciencia más que afectar, beneficia; porque el individual sólo puede ser estudiado individualmente o bien de modo aislado. Porque la experiencia de la sensibilidad no puede ser compartida, sufrida o experimentada sino por el sujeto que es al mismo tiempo motor y consecuencia, punto de partida como protagonista pero también agente del ejercicio de la sensibilidad.

Pero al mismo tiempo este autor hace de la conciencia no solamente una operación individual concreta y subjetiva, sino que reconoce que su ejercicio sólo tiene sentido si se pone en común con el otro; si se construye en la intersubjetividad como una suerte de escenario que no es único sino variable, que se evalúa y se reconsidera, que se aprueba y se desecha.

$Y$ es que una naturalización que tiene como resultado el intercambio con el entorno en última instancia no es una naturalización reduccionista sino reductiva; porque lo particular, tal como el ejercicio mental, no tiene otra 
función que formar un elemento colectivo en conjunto con el ejercicio mental de los demás.

De esta manera, a mi juicio, la naturalización de la conciencia llevada a cabo por Dennett nos permite en cierto sentido una ingeniosa combinación del estudio de lo concreto, volcado hacia lo colectivo; de lo personal, hecho construcción común. En un ejercicio que hace de la naturalización en alguna medida una desnaturalización, porque si bien las ciencias cognitivas se refieren a los fenómenos individuales operacionales cuyo efecto es la construcción global del intercambio de los ejercicios mentales, rebasan el ámbito de la experimentación homologante.

De modo que me parece más aprovechable esta ruta de la naturalización, que pone en juego una facultad concreta que al final se convierte en ejercicio colectivo; mucho más útil que una homologación de escondido corte metafísico que pretendiendo salvar la supuesta esencia de lo común, se desatiende de la individualidad del único existente: es decir, el sujeto concreto que somos cada uno. O bien, la homologación reduccionista que prefiere eliminar la referencia a lo colectivo para dirigirse exclusivamente a la experiencia individual e incomunicable.

Dennett tiene la gran virtud de haber enseñado las dos caras de un fenómeno inexplicable por el mero reduccionismo naturalista: por un lado, la peculiar entidad de una realidad que nos pertenece en exclusividad (la conciencia), pero cuyo ejercicio no es prioritariamente la introspección aislante, sino la puesta en común mediante el ejercicio de la misma, con el ejercicio de las demás conciencias; lo común, no obstante lo peculiar. Y por otro lado, la necesaria individualidad del sujeto que ejerce actos conscientes. De modo que tenemos una facultad de cada quien, para ejercerse en comunidad. Nin- 
Naturaleza y Libertad. Revista de estudios interdisciplinares. Número 4, 2014. ISSN: 2254-9668

guno de los dos aspectos de la conciencia puede tomar su explicación del sólo naturalismo, que por definición considera lo explicado como un fenómeno estandarizado, homologante, el mismo para siempre y para todos. Es decir, etéreo por común.

Así, el carácter reductivo/colectivo parece más afortunado al momento de pretender explicar una realidad de la que depende nuestra idea de grupo, de humanidad; pero también nuestra identidad como sujetos, auto determinados, auto poseídos y libres. Y en esta tarea, la propuesta naturalista de la conciencia de Dennett, creo que puede ser de singular utilidad.

\section{BIBLIOGRAFÍA EMPLEADA}

D. Dennett, Content and Consciousness, 1969 (Contenido y conciencia, Barcelona, Gedisa, 1996).

—-, Brainstorms: Philosophical Essays on Mind and Psychology, 1981

—- Consciousness Explained, 1991 (La conciencia explicada, Barcelona, Paidós, 1995).

—- Darwin's Dangerous Idea: Evolution and the Meanings, 1996 (La peligrosa idea de Darwin, Barcelona, Galaxia Gutenberg, 1999).

- - Sweet Dreams: Philosophical Obstacles to a Science of Consciousness, 2005 (Dulces sueños: Obstáculos filosóficos para una ciencia de la conciencia, Buenos Aires / Madrid, Katz Editores, 2006).

Héctor Velázquez Fernández

hvelazqu@up.edu.mx 